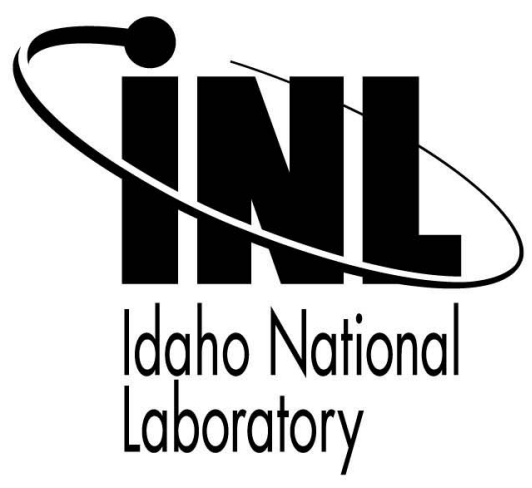

\title{
Intelligent Unmanned Vehicle Systems Suitable for Individual or Cooperative Missions
}

\section{Symposium on Defense \& Security 2007 "Unmanned Systems Technology IX"}

\author{
Matthew O. Anderson \\ Mark D. McKay \\ Derek C. Wadsworth
}

This is a preprint of a paper intended for publication in a journal or proceedings. Since changes may be made before publication, this preprint should not be cited or reproduced without permission of the author. This document was prepared as an account of work sponsored by an agency of the United States Government. Neither the United States Government nor any agency thereof, or any of their employees, makes any warranty, expressed or implied, or assumes any legal liability or responsibility for any third party's use, or the results of such use, of any information, apparatus, product or process disclosed in this report, or represents that its use by such third party would not infringe privately owned rights. The views expressed in this paper are not necessarily those of the United States Government or the sponsoring agency. 


\title{
Intelligent Unmanned Vehicle Systems Suitable for Individual or Cooperative Missions
}

\author{
Matthew O. Anderson, Mark D. McKay, Derek C. Wadsworth \\ Idaho National Laboratory \\ P.O. Box 1625 / MS 2220 Idaho Falls, ID 83415-2220
}

\begin{abstract}
The Department of Energy's Idaho National Laboratory (INL) has been researching autonomous unmanned vehicle systems for over fifteen years. Areas of research have included unmanned ground and aerial vehicles used for hazardous and remote operations as well as teamed together for advanced payloads and mission execution. Areas of application include aerial particulate sampling, cooperative remote radiological sampling, and persistent surveillance including real-time mosaic and geo-referenced imagery in addition to high-resolution still imagery. Both fixed-wing and rotary airframes are used possessing capabilities spanning remote control to fully autonomous operation. Patented INLdeveloped auto steering technology is taken advantage of to provide autonomous parallel path swathing with either manned or unmanned ground vehicles. Aerial look-ahead imagery is utilized to provide a common operating picture for the ground and air vehicles during cooperative missions. This paper will discuss the various robotic vehicles, including sensor integration, used to achieve these missions and anticipated cost and labor savings.
\end{abstract}

Keywords: autonomous, unmanned vehicle system (UVS), intelligent, cooperative, mission execution

\section{INTRODUCTION}

In operation since 1949, the Idaho National Laboratory (INL) is a science-based, applied engineering national laboratory dedicated to supporting the U.S. Department of Energy's (DOE) missions in environment, energy, science, and national defense. Currently, INL is the DOE lead lab in nuclear energy research and development. Located about 45 miles west of Idaho Falls, INL land area consists of 569,135 acres (889 square miles) as depicted in Figure 1. INL land consists of flat to gently-rolling, high-desert terrain that lies about 5,000 feet above sea level. Isolated buttes on NL reach 6,572 feet while individual mountain peaks just north and west of the site's borders approach 11,000 feet. Vast sagebrush flats dominate INL's landscape. The site lies over part of the Snake River Plain Aquifer, the largest aquifer in Idaho and one of the most productive in the nation.

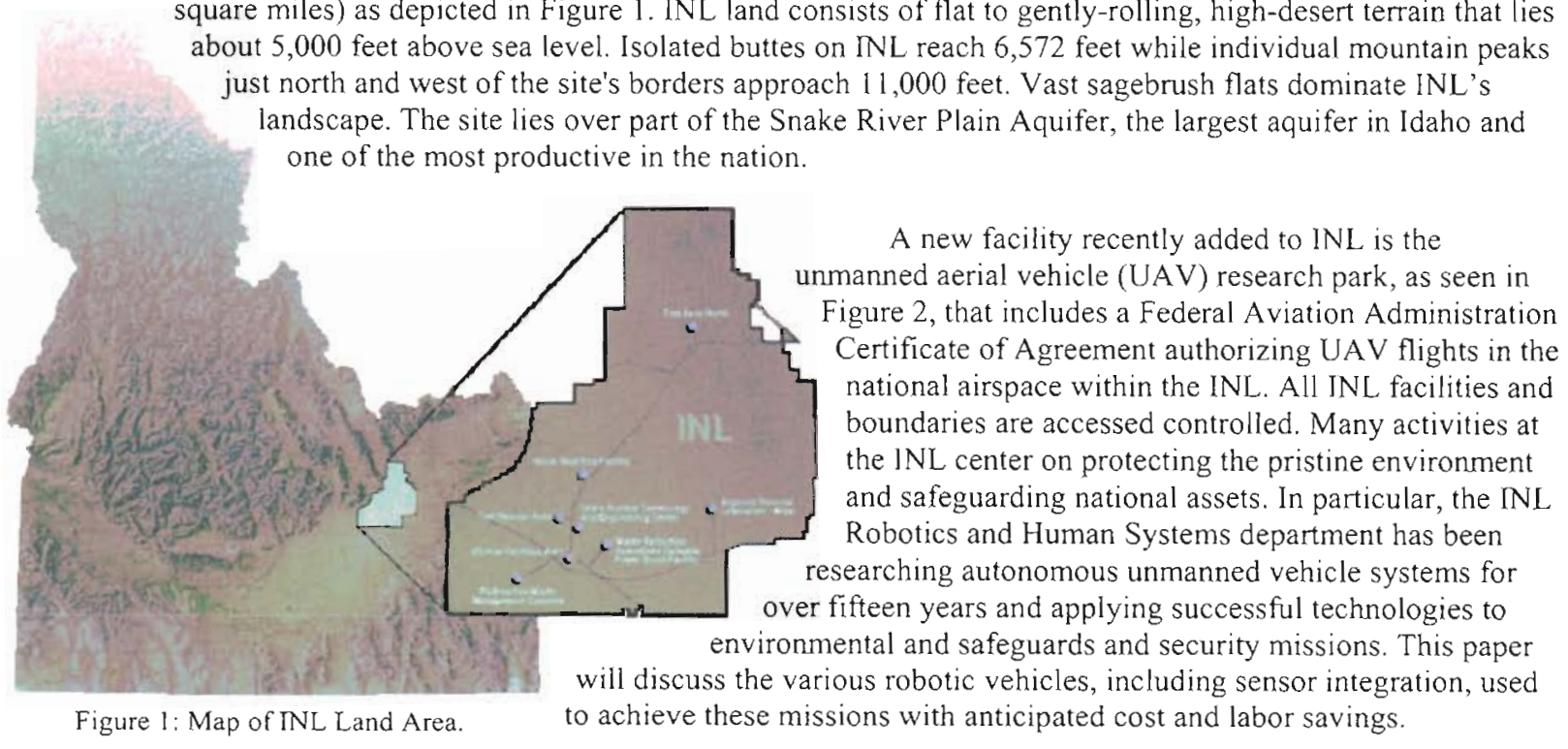




\section{1}

\section{Environmental Mission Background}

Since the 1960 s, the INL has performed radiation surveys to assess the impacts of its operations on the environment. Historically, these surveys were performed with hand-held instruments and documented with manually drawn maps. This method was time consuming and tedious, increased worker exposure to hazardous environments, and limited worker accessibility during both normal and emergency operations. State-of-the-art automation was introduced in the 1990s using a meter resolution global positioning system (GPS), MS DOS-based computers, and scintillating radiation detectors mounted on four-wheel drive vehicles. Figure 3 shows a commercially available HUMMV with a scintillating radiation detector mounted on the front of the vehicle that is typical of the equipment used to perform radiological surveys.

Instrumentation, electronics, and an operator

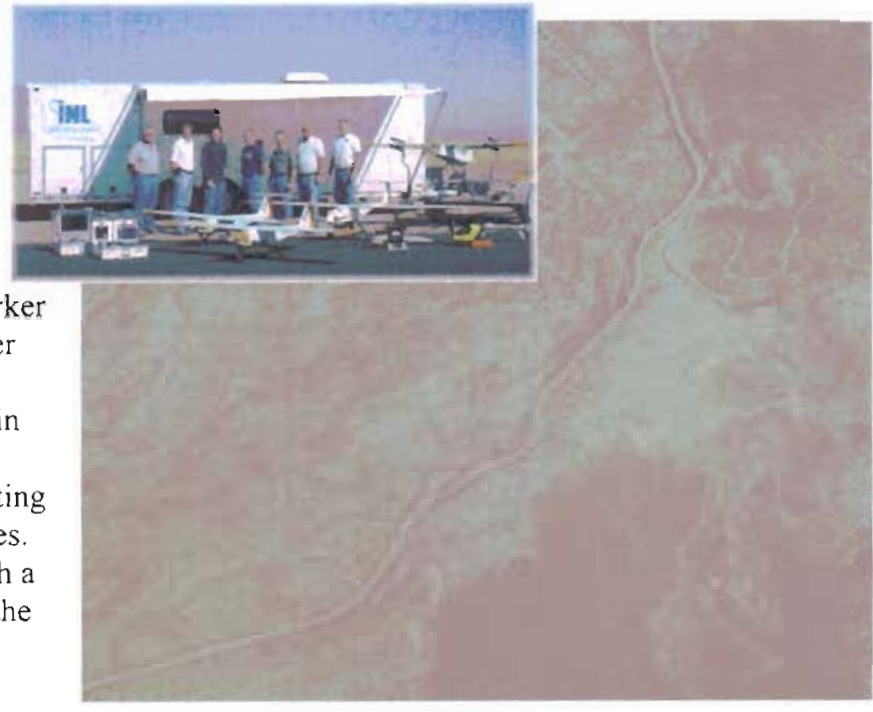

Figure 2: INL UAV Research Park with 1000' runway. control station are mounted inside the vehicle, where a human drives at 3 to $5 \mathrm{mph}$ while watching the display that reports radiation readings combined with vehicle position in real-time. A second operator is dedicated to watching for obstacles. These radiological and positional readings are stored on-board the control station and later used to generate final reports.

With the recent added emphasis on efficient and cost effective cleanup and monitoring of DOE sites, fast and accurate surveys are important for several reasons. Cost and time savings are realized by:

- Reduced manpower to perform the survey,

- More accurate and complete results,

- Reduced exposure to personnel, and

- Ability to sample in hazardous environments.

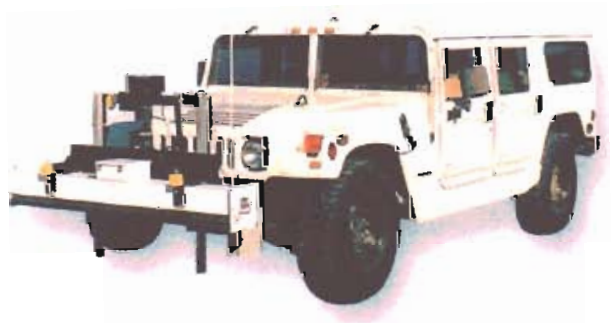

Figure 3: Manned HUMMV with scintillating radiation detector.

Continuing on through the late 1990 's and early 2000's, increasingly advanced technologies have been added to enhance the capabilities of the environmental program to achieve more effective clean-up, such as:

- Robotics,

- Sub-meter differential GPS,

- Field-hardened graphical user interface computers, and

- Autonomous parallel path swathing.

Key robotic technologies include the robotic all-terrain vehicle (RATV) and remote UAV sampling and surveillance systems used for hazardous and remote operations, as well as teamed together for advanced payloads and mission execution. Areas of application include aerial particulate sampling, cooperative remote radiological sampling, and high resolution still imagery. Both fixed-wing and rotary airframes are used, possessing capabilities spanning remote control to fully autonomous operation. Patented INL-developed auto steering technology is taken advantage of to provide autonomous parallel path swathing with either manned or unmanned ground vehicles. Aerial look-ahead imagery is utilized to provide a common operating picture for the ground and air vehicle during cooperative missions. 


\subsection{Safeguards and Security Mission Background}

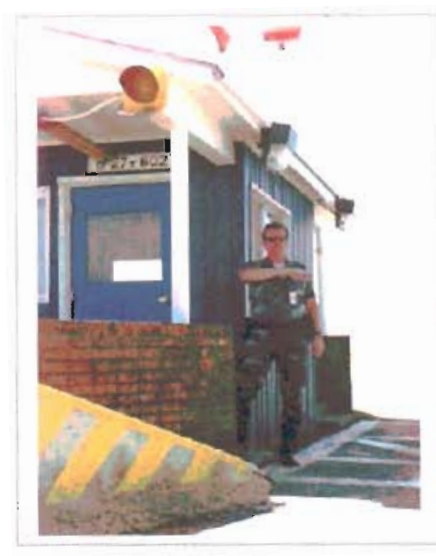

Figure 4: INL Protective Force performing safeguards and security duties.

The mission of the Safeguards and Security Program at the INL is to manage, control, and account for all nuclear material within applicable DOE and Nuclear Regulatory Commission requirements. This program provides physical protection, accountability, and nuclear material control by restricting access. This mission includes Personnel Security (limiting access to only those individuals who have the required access authorization), Physical Security (protecting facilities via access control, alarms, and barriers), and Protective Forces (uniformed guards stationed at INL facilities responsible to protect special nuclear material, personnel, government property, etc) as shown in Figure 4

World events and homeland security issues have heightened the need for surveillance and sensor deployments. At the same time, the increased number of facilities and areas which need increased security support require that reduced cost alternatives be found. In addition, technologies which reduce the burden on operators performing tedious tasks and that reduce the risk of manned operations in hazardous environments must be found. Safeguarding and securing the nations natural resources, industrial capabilities, nuclear facilities, and chemical processing sites have become an increasing responsibility. Traditional security approaches involve manned rotary wing helicopters or fixed wing aircraft to perform border patrol, provide aerial surveys, or capture and recovery of potential threats. UAVs are being developed to meet an everincreasing range of needs such as perimeter patrol, aerial surveillance, airborne communications relays, chemical or biological detection, or even as a weapons platform to detect, identify, and neutralize an enemy threat. UAVs are seen as a significant technology whose current maturity offer relevant low-cost capabilities at minimal operational and personnel risks in dull, dirty, and dangerous environments. In many situations, UAV technology can either augment or replace traditional manned aircraft.

\section{ROBOTIC GROUND TECHNOLOGIES}

\subsection{Robotic All-Terrain Vehicle}

The RATV is built on a commercially available hydrostatic eight-wheel drive amphibious vehicle platform and was made into a robotic vehicle by Utah State University in the late 1990's.' Early applications of the RATV included agriculture missions. ${ }^{2}$ This platform was further modified to deploy a scintillating radiation detector mounted on the front and a highresolution gamma spectrometer

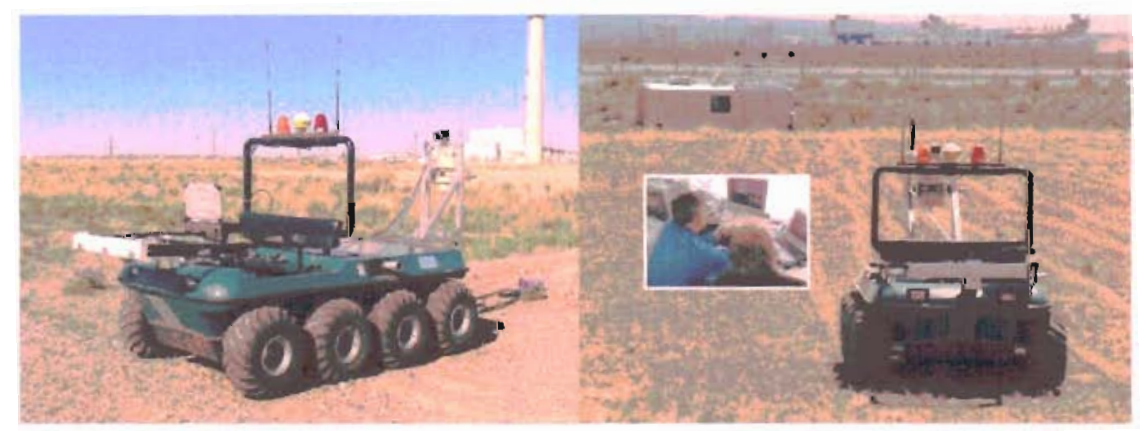

Figure 5: RATV being operated in the field relaying data to the remote control station. mounted on the rear of the vehicle. It also has a control computer that accommodates manual, remote, or fully autonomous operations. Data from the scintillating radiation detector and gamma spectrometer can be relayed to a remote control station in real-time for full remote operations. The RATV was modified with sub-meter differential GPS and field-hardened graphical user interface computers. It is also programmed for manual and autonomous control of parallel path swathing that minimizes "skip" and "overlap" while performing track-to-track passes using patented INL-developed auto steering technologies." This is a necessary and vital capability for cost-effective operations. Figure 5 shows the RATV being operated autonomously in the field relaying radiological and position data to the remote control station. 
The RATV has been used to survey areas that were historically surveyed manually. These surveys were performed at the INL's Test Reactor Area (TRA), Test Area North (TAN), and the Auxiliary Reactor Area (ARA). In each of these events, data from the scintillating radiation detector is directly imported into specialty software and maps, and can be quickly prepared to report survey results to state regulators. As well as being used to perform routine surveillance and screening activities, the RATV can also be used to characterize and remediate contaminated areas by evaluating specific isotope concentrations in surface soils. This is accomplished by taking field measurements using the scintillating radiation detector and the in-situ gamma spectrometer in contaminated areas within sites of concern. Gross counts are compared to the insitu measurements and graphed. A regression is performed to develop an equation that converts counts per second to isotopic concentrations. Areas where target cleanup levels have been met are easily identified. Subsequent remote surveys show the

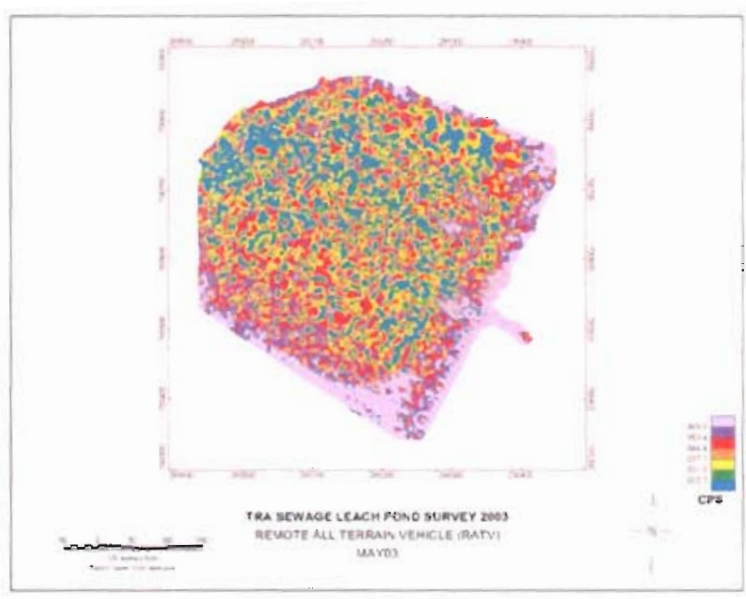

Figure 6: Test Reactor Area survey results. progress of remediation efforts and identify areas where additional work is required to achieve remediation goals. Figure 6 shows a typical survey performed using the RATV platform in this manner. ${ }^{4}$

\subsection{Autonomous Robotic Countermine System Unmanned Ground Vehicle}

\section{The Autonomous \\ Robotic Countermine System (ARCS) project is a cooperative effort to provide behaviors which enable teams of small unmanned ground vehicles (UGVs) and UAVs to collaboratively conduct semi-autonomous countermine operations in live and virtual environments. The participants in this project include the INL, Carnegie Mellon University (CMU), the Space and Naval Warfare Systems Center San Diego (SPAWAR), and the U.S. Army Maneuver Support Center (MANCEN) Futures Center. The U.S. Army Night Vision and Electronic Sensors Directorate at Ft. Belvoir, Virginia also provided significant input. The} equipment developed and integrated for the experiment includes a robotic platform, a mine sensor payload, a dye marking package, the INL Robotic Intelligent Kernel (RIK), ${ }^{5}$ and an operator control unit. Figure 7 shows the ARCS UGV.

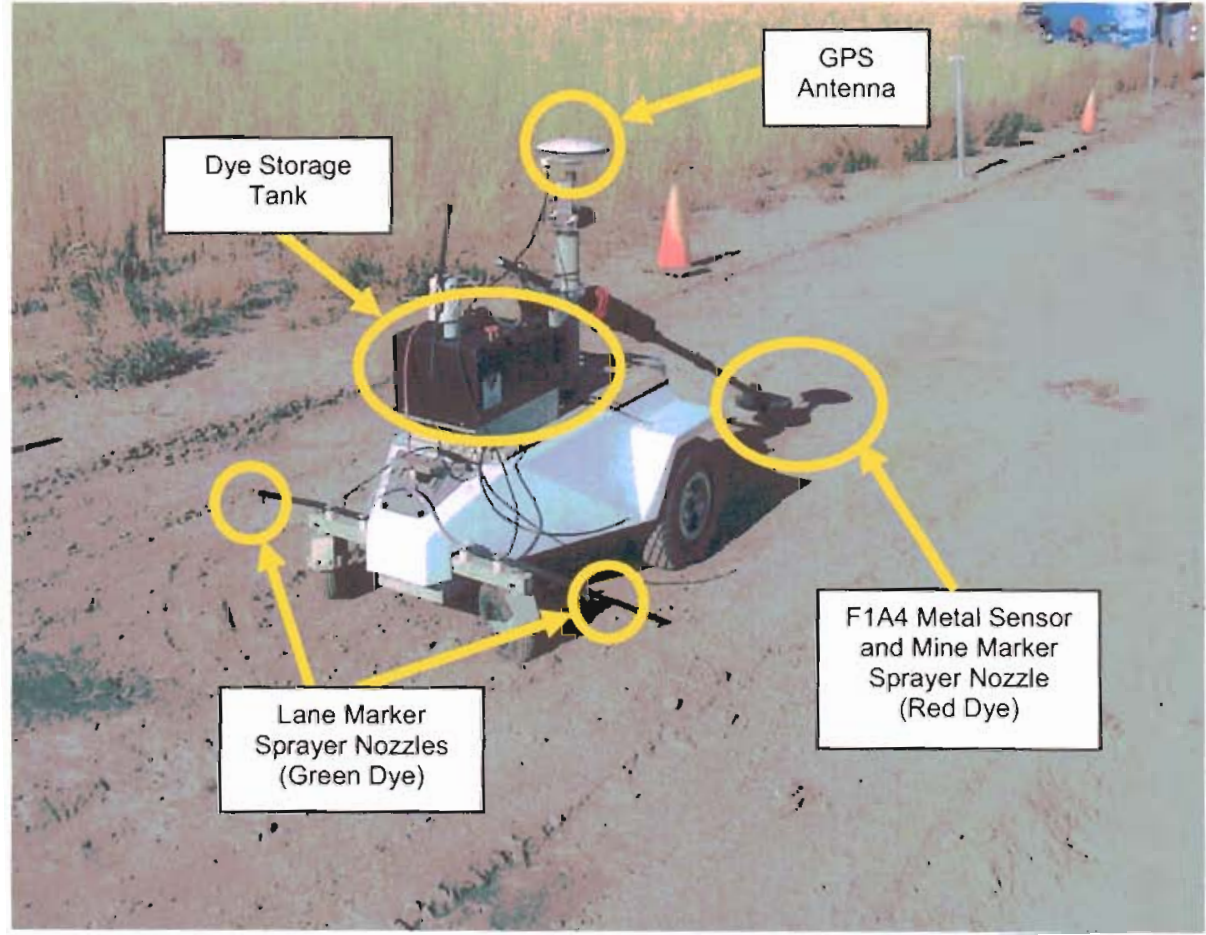

Figure 7: The ARCS UGV. 
The ARCS UGV is from the Future Combat Systems Learning Applied to Ground Robotics (LAGR) robot developed by CMU. Two robots were built and modified by CMU and delivered to INL for integration. The modifications to the robot include a mechanism and control package for the mine sensor. This mechanism provides a mount for the MineLab F1A4 metal detector, which is the standard issue mine detector for the U.S. Army. This mechanism provides a height adjustment and pivot system for sweeping the mine sensor over the ground, and a torque sensor to recognize when the sensor is touching the ground. Included is the software control system for the robot platform and sensor payload that are linked to the RIK. The dye marking system was designed and built by SPAWAR and is mounted on the rear deck of the robot. The marking system consists of two dye tanks; a larger one for marking the cleared lane, and a smaller one for marking the mine location. The system also includes pumps, hoses, and nozzles for dispensing the dye, and a control system that links to the RIK.

The RIK and operator control station were developed at the INL and were extended to provide a plug and play mine detection and marking capability to any platform that uses the RIK and can carry a countermine sensor payload. ${ }^{5}$ The operator control station was modified to recognize when the countermine capability is available on the robot and provide the appropriate tasking options. Additionally this control station was used in a collaborative UAV to UGV effort to create a common operating picture for the ARCS operator. This will be described in further detail in this paper.

\section{ROBOTIC AERIAL TECHNOLOGIES}

In addition to the work being performed with the UGVs, remote aerial sampling and surveillance equipment is being developed and tested at the INL using both fixed-wing and rotary vehicles. INL has been involved in UAV research for the last decade, developing specific UAV capabilities and behaviors under INL research and development programs, as well as Defense Advanced Research Projects Agency (DARPA) programs.

\subsection{Fixed-wing UAVs}

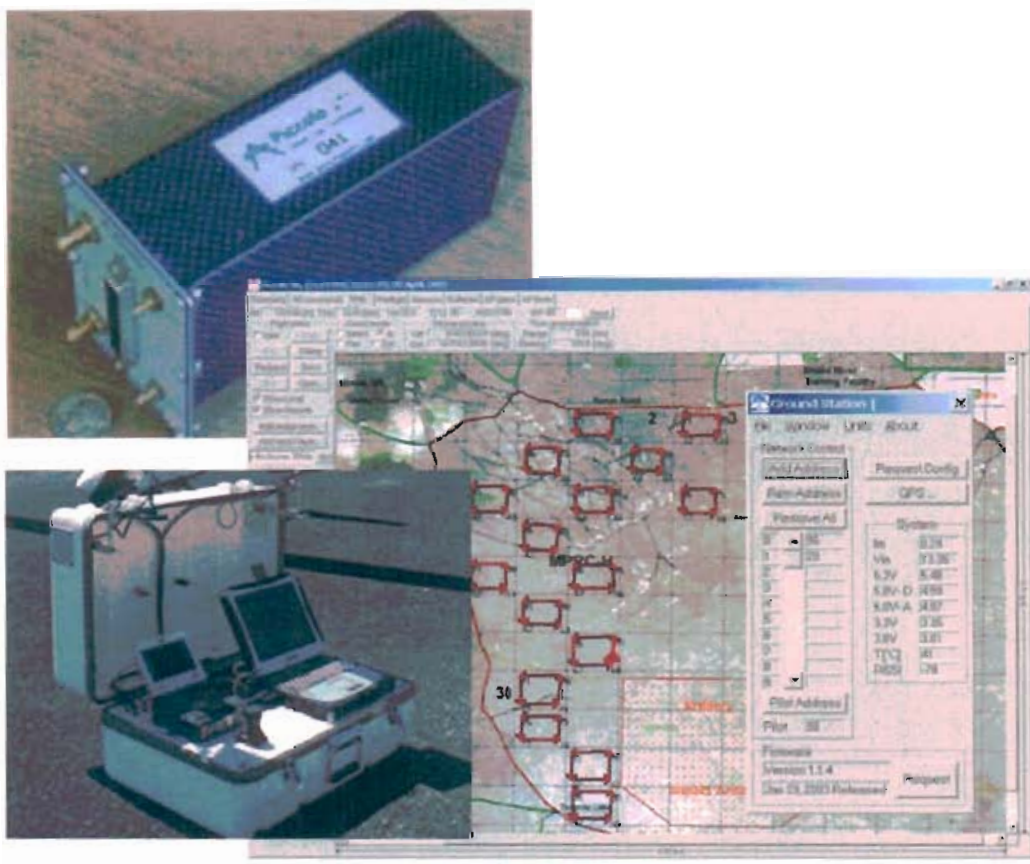

Figure 8: UAV Autopilot and portable ground control station.
The INL is engaged in the development, testing, and evaluation of portable UAV technologies. A fixed wing airframe and subsystems have been integrated and deployed in a variety of configurations with maturing missions. A driving factor in all technologies developed is the best use of affordable technology. UAV airframes have been designed and built with the performance and payload targeted at the mission and software developed to augment a commercial flight autopilot and ground control computer. The payload includes a stabilized live visual video feed and communications are provided by line-ofsite radio frequency communications. Numerous industry partners are involved in the INL fixed-wing UAV development including two key partners - Cloud Cap Technology and Arcturus UAV.

At the core of the UAV program is the Cloud Cap Technology Piccolo II autopilot. The Piccolo Il avionics package provides the capabilities of a high-end autopilot at a significantly lower cost. A standard autopilot system consists of the Piccolo II avionics and a ground control station (see Figure 8). 
Key features of the autopilot system include:

- Programmable GPS waypoint navigation,

- Altitude and airspeed hold,

- Auto Assist flight modes,

- Full integration with 3-axis gyros, accelerometers, GPS receiver, and pressure sensors,

- Integrated $900 \mathrm{MHz}$ RF link,

- Ground and in-flight programmable flight plans,

- Parachute / light / brake control,

- Programmable flight termination or return to base due to loss of communications or system failure, and

- Access to flight controls via secondary serial interface.

Included with the autopilot system is ground-control software that offers the user a point and click interface for mission planning that runs on a Windows-based computer. If lineof-sight control is maintained, the software allows the operator to monitor and log vehicle telemetry data, change waypoints, upload new flight plans, initiate holding patterns, and adjust feedback loops, all while the UAV is in flight. ${ }^{6}$

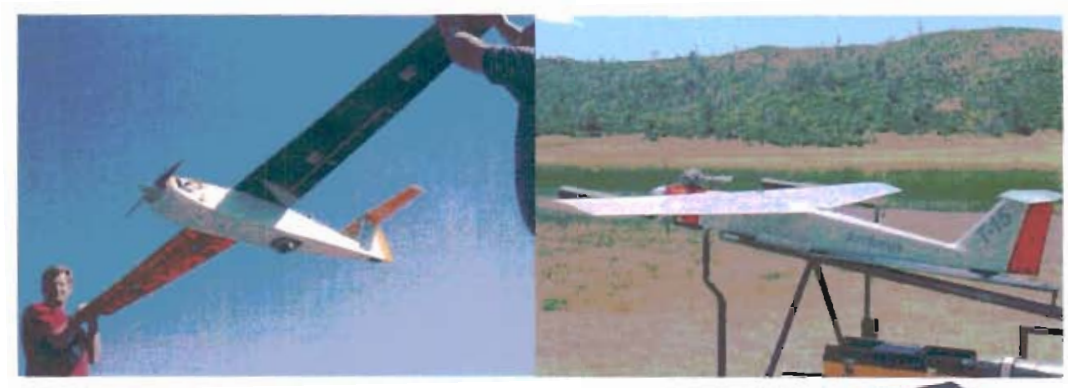

The primary fixed wing platform is the Arcturus UAV T-15 airframe. The T-15 is a composite molded, high performance UAV. It was specifically designed to fill the niche for a portable, medium endurance ( 12 hours) vehicle capable of a 10 pound payload capacity. The T-15 is a very stable UAV and can be pneumatically launched for full autonomous flight from start to finish. ${ }^{7}$ Features of the T-15 include:

- Honda GX-50 $50 \mathrm{cc} 4$-stroke engine,

- $10^{\prime}$ wing span,

- 6 ' length,

- 1440 square in. wing area,

- 45 lbs. maximum weight,

- 28 lbs. dry weight,

- Maximum speed of 90 knots,

- Cruising speed of 50 knots,

- Stall speed of 27 knots,

- 10 lbs. payload capacity, and

- Endurance period of 12 hours.

The T-15 is outfitted with a stabilized gimbaled video system weighing approximately $32 \mathrm{oz}$. with a 4.4 inch diameter turret as seen in Figure 9. This camera system is fully integrated with the Piccolo II autopilot allowing either manual control or full automated "sensor point of interest" control that keeps the commanded Latitude /

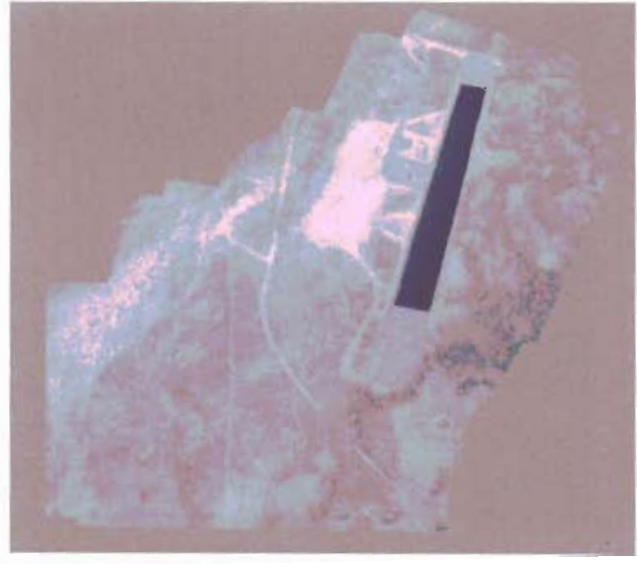

Figure 10: UAV real-time mosaic imagery of the INL, UAV runway.

Longitude / Altitude point of interest in continuous view, while the UAV traverses the commanded flight path. Analog imagery from the UAV is relayed to the ground station for direct referencing of the image on geo-referenced maps or to be viewed as a real-time mosaic image as shown in Figure 10. This is accomplished using a customized application working in concert with a Pyramid Vision Technologies Acadia I Vision Accelerator PCl board on a PC. ${ }^{8}$ 


\subsection{High Altitude Air Sampler}

The High Altitude Air Sampler (HAAS) is a small UAV designed for collecting real-time air samples of airborne plumes, such as those occurring during wildfires or catastrophic events. The HAAS utilizes state-of-the-art technology for accurate real-time air quality and air contaminate characterization. HAAS is a small, lightweight, portable continuous air monitoring system, suitable for collecting a particulate sample of $300+$ cubic feet of air.

The HAAS UAV eliminates the risk to personnel and equipment during wildfires or catastrophic events when accurate and real-time sampling is needed. HAAS has been calibrated against the standard continuous air monitoring systems currently used by the INL Environmental Services Program. Proper real-time monitoring requires that sensors be placed in the plumes where extreme dangers such as turbulence, contamination, and zero visibility make ground human entry impossible. Current operations place land-based monitors at a distance of 2 to 5 miles from the source. These landbased sensors collect only a small representation of the plume. While it is possible, the operation of man-piloted aircraft into a fire/smoke plume presents extreme danger to the pilot and the aircraft and unwarranted risk. Thus, a system to deploy monitoring and sampling sensors to measure real-time conditions in airborne plumes is a real need.

The HAAS is based on a commercially available Rascal radio control (RC) airframe. The airframe has been modified for autonomous operation and greater lift/payload capacity and longer flight duration. In the current configuration, the HAAS UAV has a flight time of 1 hour and the air sampler can be remotely activated and operates for up to 30 minutes. The engine exhaust has been routed away from the air sampler filter intake to minimize particulate contamination. The UAV utilizes the Piccolo Il autopilot. Typical flight altitude is $1000 \mathrm{ft}$ above ground level (AGL), but it can vary anywhere from $100 \mathrm{ft}$ and up. The air sampling system is based upon a commercially available impellor and motor system powered by lithium polymer batteries, an electronic speed controller, and INL-developed electronics for on-board data storage and real-time monitoring at the UAV ground control station. Figure 11 shows the HAAS emphasizing the sample air intake system.

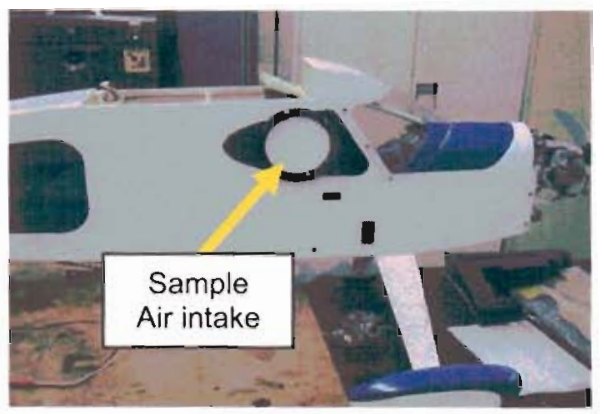

Figure 1 I: INL High Altitude Air Sampler.

On May 25, 2004, the first test flight was conducted at the INL UAV research park. The wind conditions ranged from 5 to $10 \mathrm{mph}$. The flight consisted of an oval pattern and lasted approximately 1 hour. The total sample time was 33 minutes with an accumulated sample of 328.1 cubic feet of air. At about 5 minutes into the flight, the autonomous controller was activated. At about 12 minutes, the sampler was activated. No anomalies were observed during the flight, and all of the systems performed normally. The flight was successful and demonstrates the capability for real-time plume sampling. The next step is to flight-test during a controlled burn and collect samples from the smoke plume.

\subsection{Rotary UAV}

\subsubsection{Radio controlled rotary UAV}

For the past several years, small RC helicopters with flight stabilization have been used for collecting aerial imagery. A minihelicopter camera system dubbed the "miniheli-cam" was designed for quick transport and deployment and ease of operation (see figure 12). With only a 53-5/8 inch diameter rotor and weighing less then 8.5 lbs., the mini-heli-cam is capable of stable flights of up to $1000 \mathrm{ft}$. AGL. The ground receiver can completely fit into a

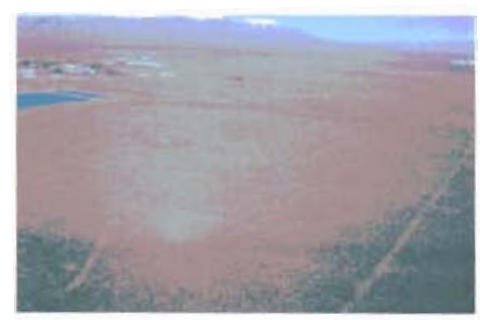

Figure 13: Aerial imagery of wastewater treatment plant from rotary UAV. small briefcase and one person can easily transport the entire system. The camera shutter is operated from the RC joystick transmitter and the camera image can also be recorded to tape. The small camera is capable of capturing 248 4-mega pixel images. Figure 13 shows high resolution still imagery taken from a rotary UAV. 


\subsubsection{Autonomous rotary UAV}

The AutoCopter is a selfstabilized mini-helicopter developed by Neural Robotics Incorporated (NRI). It weighs $45 \mathrm{lbs}$. and measures $2.5^{\prime} \times 2^{\prime} \times$ 6' ( $\mathrm{H} \times \mathrm{W} \times$ Rotor Diameter). The AutoCopter uses a proprietary neural network controller to stabilize and fly the system. The AutoCopter can maintain stable flight and accurate position in the presence of external disturbances such as winds or a swing load. One unique feature is that it doesn't require a lot of flight training or flight experience. Using just the RC joystick transmitter, a pilot can maneuver forward, backward, sideways, and up or down. With the use of a laptop computer, the AutoCopter can fly to GPS waypoints or a grid. The $\mathrm{PC} / 104-$ based avionics consists of an attitude and heading reference, a WAAS (wide area augmentation system) compatible GPS, an ultrasonic altimeter, a barometric pressure sensor, a heading hold gyro, and a CPU. Currently the

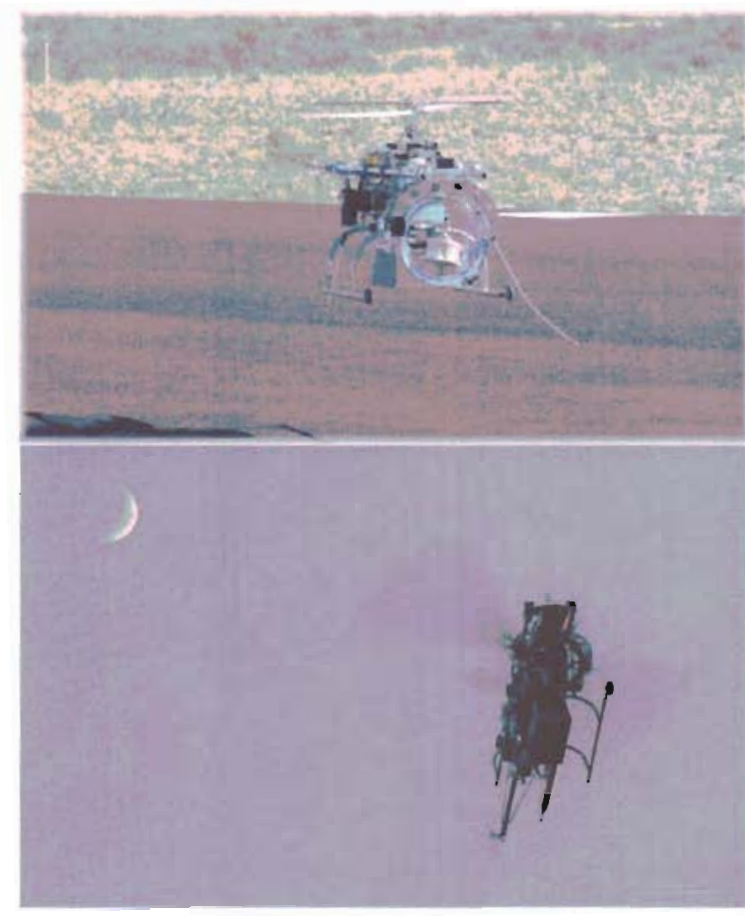

Figure 14: NRI Autocopter in flight at INL UAV research park.

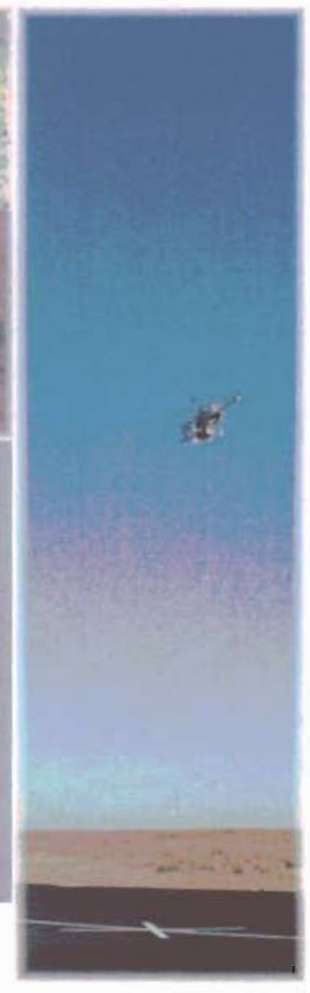

AutoCopter is capable of operating

within a 1.5 mile radius of the transmitter. In the event of loss-of-communications, the unmanned helicopter will automatically return to the launch point. The AutoCopter is capable of completely autonomous launching and landing as shown in Figure 14.

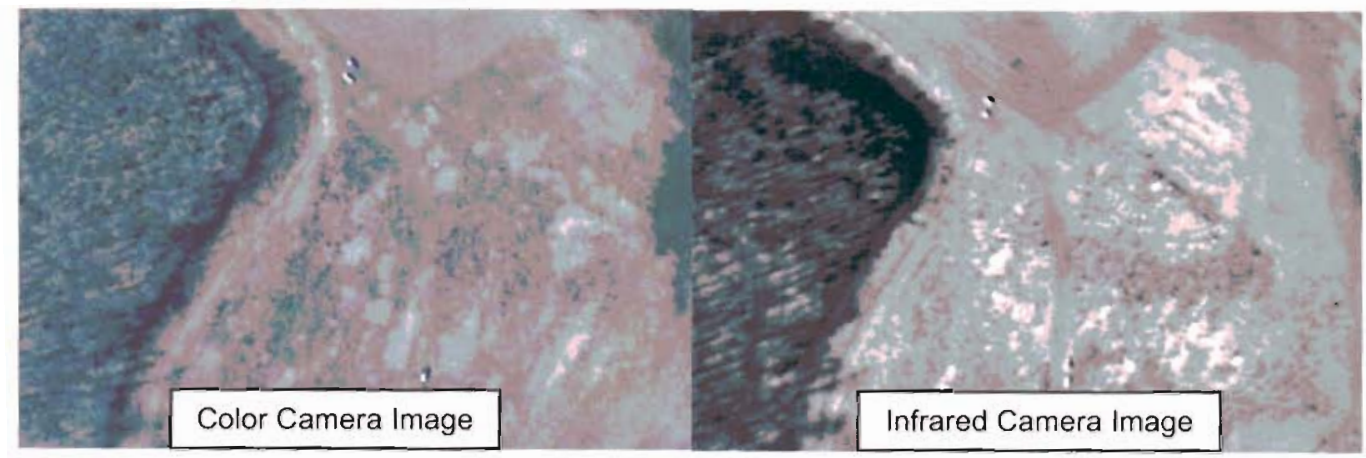

Figure 15: Thermal imagery from the Autocopter.

The standard NRI system is equipped with a 3-axis gyro stabilized camera platform. The platform can be adjusted to hold a payload of up to $15 \mathrm{lbs}$. The platform can also be "fixed" to accommodate applications requiring solid mounted sensors. The current flight time is 30 minutes but payload can be traded for additional fuel. In upcoming cooperative ground and air activities, the NRI AutoCopter will be used to demonstrate the use of manned/unmanned/ radio control/autonomous agents to perform facility protection. Figure 15 shows imagery taken from the AutoCopter. 


\section{COOPERATIVE TECHNOLOGIES}

\subsection{Cooperative UGV and UAV Environmental Monitoring}

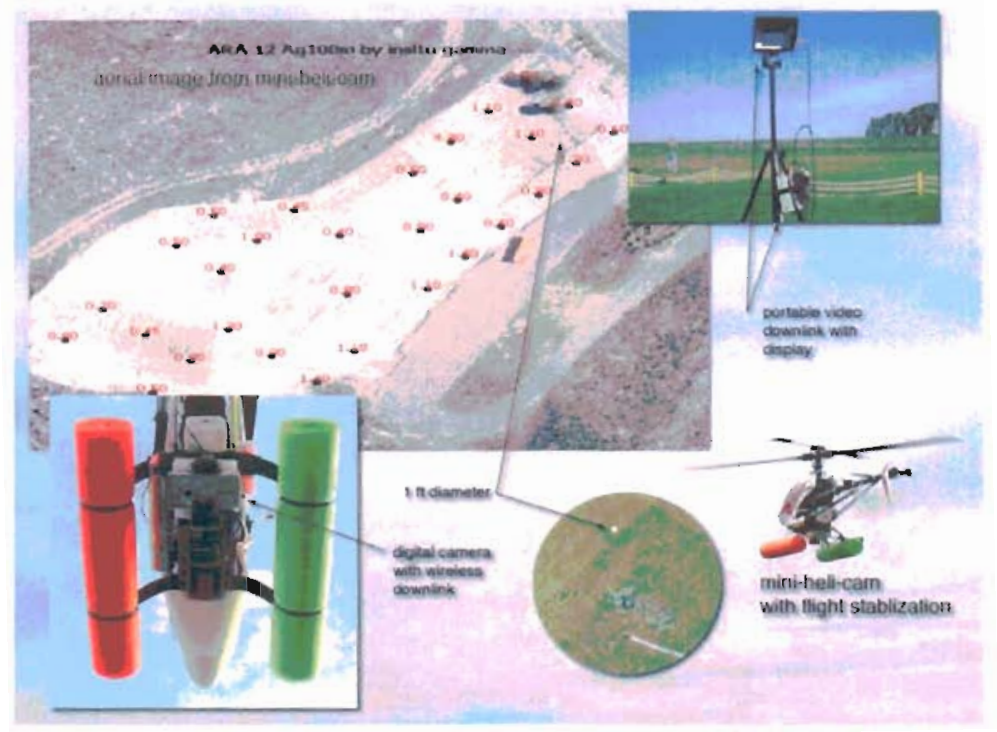

Figure 16: Resulting ARA survey with readings overlaid on aerial imagery.
During decontamination and deactivation activities at the INL, it is necessary to characterize areas slated to be released into the environment. One of these identified areas is the Auxiliary Reactor Area. The radiological survey required the RATV to automatically drive across the target area at 3 to $5 \mathrm{mph}$ gathering data from the scintillating radiation detector and determining counts per second (CPS) readings. At elevated reading locations, or locations that historically were contaminated, the RATV was required to position and hold the germanium detector for approximately 25 minutes to perform in-situ isotopic identification activities. During these remote operations the mini-heli-cam was used to provide the RATV operators with real-time overview imagery and critical situational awareness. At the conclusion of the survey, a high-resolution image taken from the mini-heli-cam was used to overlay

the resulting isotopic data as seen in Figure 16. This technique proved to be extremely effective and subsequent surveys were requested to be performed in this manner.

During these cooperative surveys, significant cost and time savings are realized due to a reduction in the number of workers needed to perform the survey. Beyond reducing worker exposure, project managers are able to obtain accurate and complete field data in hazardous environments. In addition to the obvious cost savings shown in the above routine surveys, the RATV / UAV team can also save time spent screening areas to reduce the number of in-situ surveys required (a very labor intensive activity that usually takes several workers all summer to perform by hand). Robotic platforms can quickly locate areas of elevated radioactivity that can be further investigated using in-situ gamma spectrometry to verify contamination levels. The robotic platforms have many unique benefits and successful results that contribute to improve surveys, including:

- Providing accurate data before, during, and after an incident for complete results,

- Reducing exposure and increasing worker safety,

- Taking samples in remote, inaccessible, or hazardous environments,

- Reducing environmental impacts and operating costs,

- Reducing fire hazards associated with conventional survey vehicles,

- Minimizing interference with remediation efforts, and

- Providing real-time video photography.

\subsection{Cooperative UGV and UAV ARCS Countermine Experiment}

In October 2005, the U.S. Army Test and Evaluation Command (TECO) and MANCEN conducted a countermine experiment at the INL UAV research park of the ARCS. The purpose of the experiment was to test the effectiveness and capabilities of ARCS, which included obstacle avoidance, path planning, terrain mapping and localization in addition to mine detection and reporting. The experiment evaluated the effectiveness of ARCS to prove a 1 -meter lane by finding mines, marking the mine location on the ground, reporting the mine location to the operator 
control unit (OCU), and marking the boundaries of the cleared lane. As part of the experiment a demonstration of UAV-UGV cooperative behaviors was presented. The demonstration consisted of the following scenario:

- A T-15 UAV was deployed to survey the terrain surrounding the airstrip.

- The real-time geo-referenced imagery from the UAV was analyzed and a possible minefield was detected.

- This mosaic imagery was geo-referenced to form a backdrop and imported seamlessly into the ARCS oCU.

- The aerial imagery was correlated with the ground robot terrain map to allow for efficient tasking and monitoring of the ARCS UGV.

- The ARCS was deployed to the minefield.

- The ARCS detected and physically and digitally marked mines.

- A proofed lane was marked through the minefield.

Figures 17 and 18 show pictures taken during the ARCS experiment and a geo-referenced mosaic image overlaid on the OCU user interface. The mosaic image was acquired via the Pyramid Vision Technologies system and required approximately 5 minutes for acquisition and geo-referencing using standard geospatial tie-point techniques.

The rigorous 10 day test showed dramatic results for this unique unmanned system. Highlights of the results showed:

- 124 of 131 inert mines detected $(95 \%)$ with 1 false positive.

- All mines detected were correctly marked on the ground and in the operator interface.

- Proofing and marking a 50 meter lane took 5 to 6 minutes, compared to approximately 24 minutes for a soldier in training to accomplish the same task

- The overall operation required less than $1 \%$ human involvement.

- The overall Autonomy Levels for Unmanned Systems (ALFUS) rating for the ARCS was calculated to be approximately 7.5 to 8 out of 10 . $^{9}$

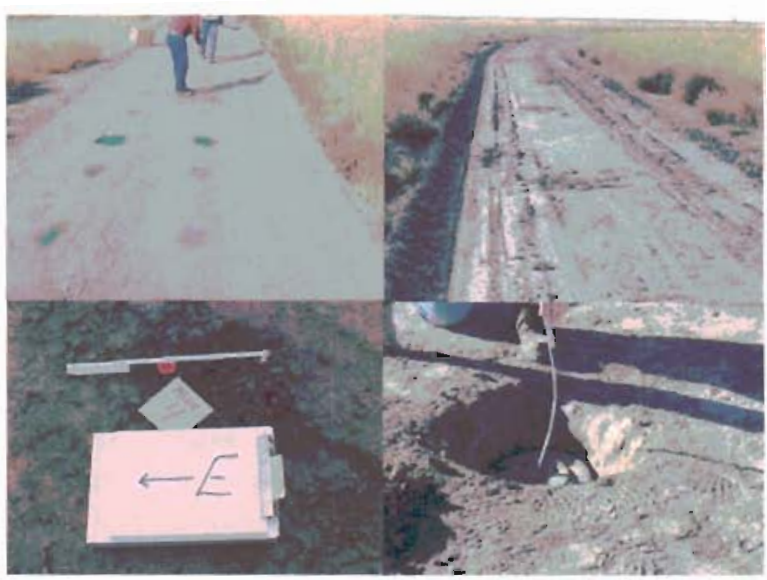

Figure 17: Buried mine lane, marked cleared lane, and samples of buried AT mines taken during the experiment.

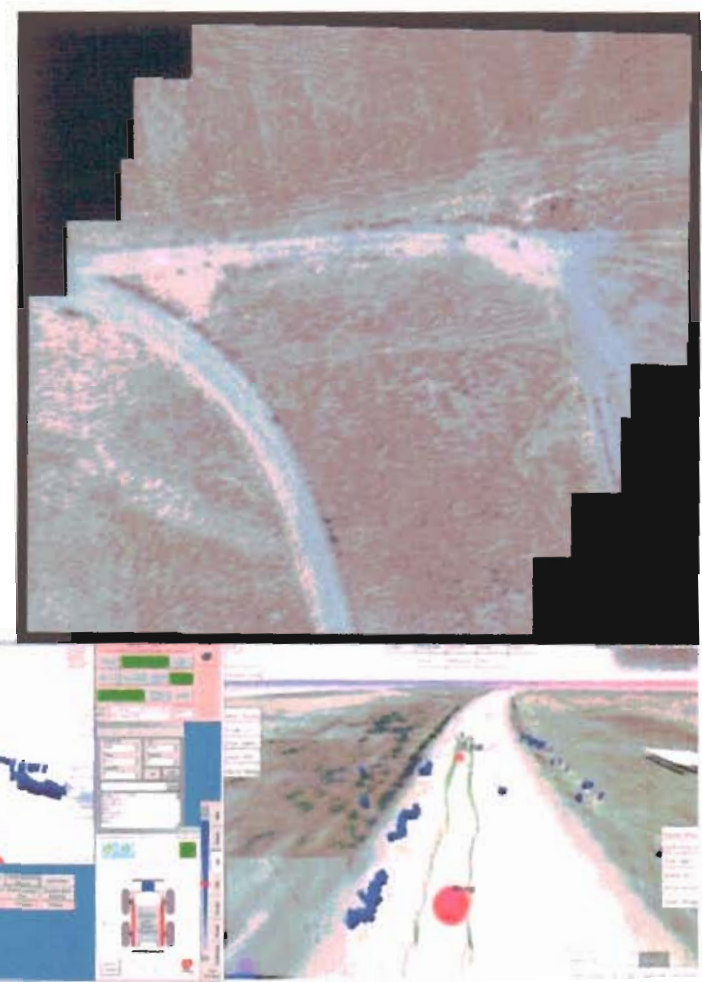

Figure 18: ARCS OCU user interface and geo-referenced mosaic image taken from the T-15 UAV.

\subsection{Cooperative Safeguards and Security Technology Demonstration}

In a joint effort between the INL UAV program and the Safeguards and Security Department, INL UAV and robotics engineers are cross training security guards on the unique skill set needed to fly UAVs. The project goes beyond the traditional research and development role that a national laboratory usually plays by incorporating the research and design of small UAVS with an element of testing and evaluation for multiple end users. Lieutenant Bob Adolfson, a 19 year veteran of the INL's security force (shown in Figure 19 during training exercises), was recommended to be the first 
security guard to undergo flight school due in part to his experience as an RC pilot. Last year, Adolfson was assigned nearly full-time to the UAV program, spending numerous hours on the runway watching, learning, and test flying the small planes. He also spent time in INL's engineering labs learning how the mechanical components, sensors, and camera systems functioned together to provide constant communication between the UAV and ground station, real-time video footage, and instant command and control options.

\subsubsection{Cooperative UAV-Protective Forces Demonstration}

Last July, INL security and DOE headquarters personnel came to the laboratory to witness first hand the security advantages offered by the use of lightweight, man-portable UAVs capable of performing real-time mission planning and persistent surveillance. As part of the demonstration, a UAV with a preprogrammed flight path was sent airborne to collect video feeds directly above the UAV runway. At the same time, several miles away, Adolfson transferred control of the plane via a mobile UAV ground station, and changed the flight path to collect footage over a different area of interest. During the two-hour demonstration, the UAV provided constant, real-time video footage from the air. In order to comply with Federal Aviation Administration's guidelines, line of sight was constantly maintained with the plane during the demonstration. Additionally, during a force on force exercise demonstrating facility and perimeter detection and protection, the UAV was used to cooperate with the ground security force. The UAV was tasked with identifying intruders, following and assessing the situation including simulated barrier explosions, and final battle damage and simulated kill assessments. Figure 20 shows pictures from the UAV and a combined presentation briefing display. ${ }^{10}$

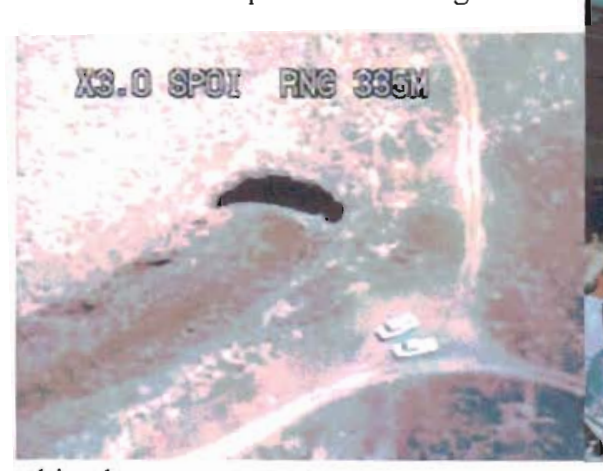

Figure 20: UAV aerial imagery during force on force exercise and VIP display.

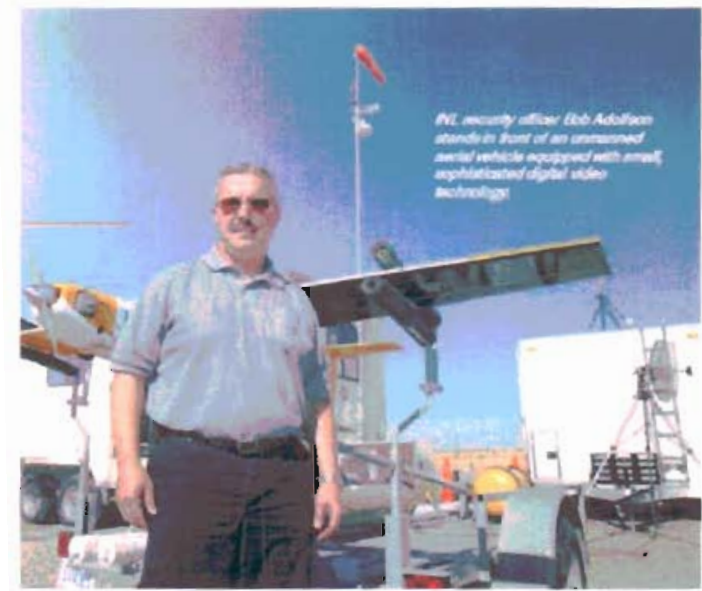

Figure 19: TNL Safeguards and Security Lieutenant Bob Adolfson undergoes UAV flight training.

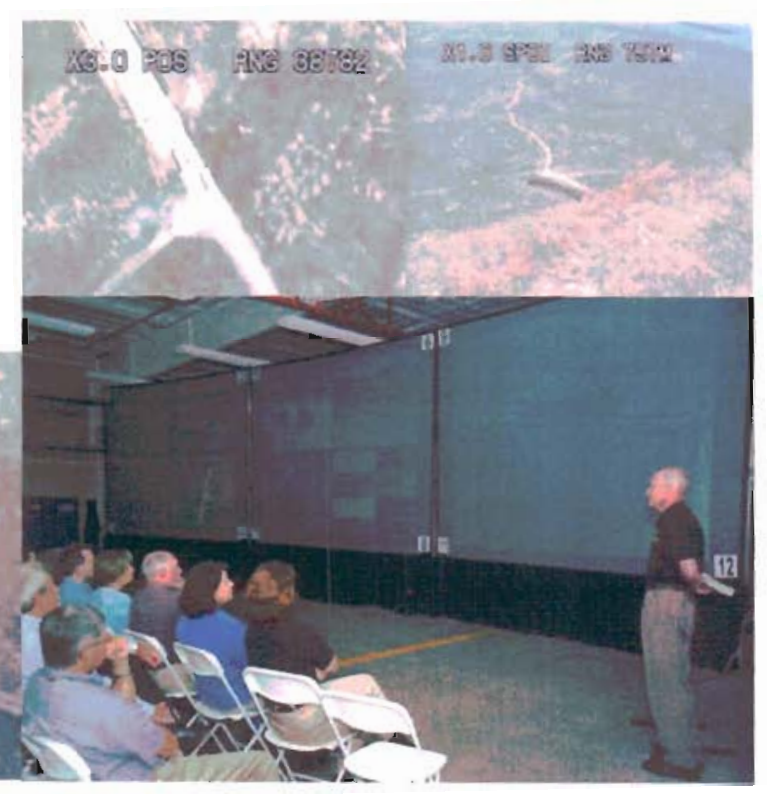

\section{CONCLUSION}

The results of the environmental monitoring / ARCS experiment / safeguard and security cooperative UVS demonstrations showed success in all goals for UGVs, UAVs, and manned assets. Robotic systems have been shown to be extremely effective in performing manual or autonomous radiological surveys, reliable in identifying and marking land mines with greater efficiency than conventional methods, and improving security operations via collaborations between man and UVS resources.

As unmanned vehicle systems become more accepted by end users, the technology baseline will move forward. As a result, the UVS tools will need to be user-centric and easy to use. This will make the technology more readily 
available to end-users, thus increasing the INL's and other DOE laboratories' core capabilities to better serve the public's needs. This is a great step in the right direction; however, additional development is being worked in the following areas:

- Integration of advanced technologies for obstacle detection and avoidance allowing safe operations during unattended autonomous modes.

- Improved user application software allowing adjustable autonomy operations.

- Cooperative intelligent geospatial path-planning and enhanced navigation capabilities.

- Development and integration of chemical contaminant detection capabilities.

- Cooperative aerial and ground scintillating radiation detector surveys.

- Improved common operating picture and data fusion for optimal situational awareness and command and control.

Developing these capabilities will advance the technology into the forefront of intelligent cooperative robotics. These state-of-the-art technologies will help DOE accomplish its missions in environment, energy, science, and national defense, as well as to protect the general public in a safe and timely manner. Given these innovative technologies, it's easy to see why the INL's robotics program leads the DOE laboratories in combining science and robotics to develop high-tech instruments to better monitor the environment as seen in Figure 21 . Among all national labs, the INL is one of the few that has the experience and expertise necessary to create next-generation technologies that will make the world a cleaner and safer place.

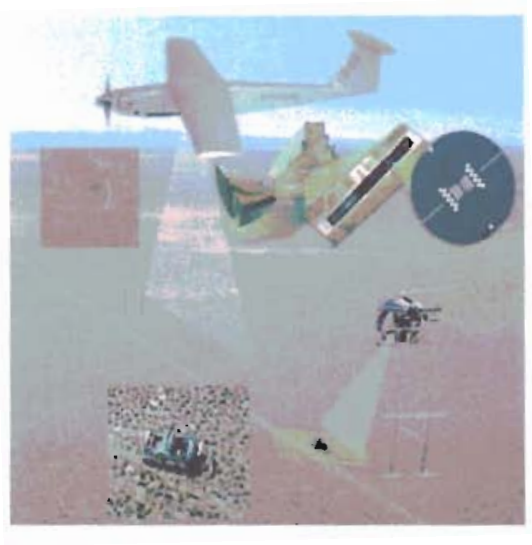

Figure 21: INL UVS technologies in real world missions.

\section{REFERENCES}

I. J. S. Jacob, R. W. Gunderson, and R. R. Fullmer, "Conversion and Control of an All-terrain Vehicle for Use as an Autonomous Mobile Robot", Proceedings of SPIE Robotic and Semi-Robotic Ground Vehicle Technology, Gerhart \& Abbott, Vol. 3366, pp. 83 92. SPIE, Orlando, FL., April 15-16, 1998.

2. M. D. McKay, M. O. Anderson, R. W. Gunderson, N. Flann, and B. Abbott, "Multi-Agent Cooperative Systems Applied to Precision Applications", Proceedings of SPIE Robotic and Semi-Robotic Ground Vehicle Technology, Gerhart \& Abbott, Vol. 3366 , pp. 108-113, SPIE, Orlando, FL., April 15-16, 1998.

3. M. D. McKay, M. O. Anderson, D. C. Wadsworth, "Development of the Auto-Steering Software and Equipment Technology (ASSET)", Proceedings of the SPIE Unmanned Ground Vehicle Technology V, Gerhart, Shoemaker, \& Gage, Vol. 5083, pp. 183-191, SPIE, Orlando, FL., April 22-23, 2003.

4. M. A. Verdoorn, M. O. Anderson, M. D. McKay, D. W. McBride, and C. P. Oertel, "Development of Autonomous Unmanned Vehicles for Large Area Radiation Surveys and Sampling," $10^{\text {th }}$ International Conference on Robotics and Remote Systems for Hazardous Environments, Gainesville, FL., March 28-31, 2004.

5. D. J. Bruemmer, M. O. Anderson, M. D. McKay, et al. "Intelligent Autonomy for Remote Characterization of Hazardous Environments," Proceedings of the IEEE International Symposium on Intelligent Control, Houston, TX., October 5.8, 2003.

6. Cloud Cap Technology homepage, http://www.cloudcaptech.com/default.htm.

7. Arcturus UAV T-15 webpage, http://www.arcturus-uav.com/t15.html.

8. Pyramid Vision webpage, http://www.pytamidvision.com.

9. M. C. Walton, D. J. Bruemmer, D. A. Few, and C. W. Nielsen, "Developing an Intelligent and Integrated Unmanned Ground Vehicle System: A Case Study," AUVSY's Unmanned Systems North America 2006 Conference. Orlando. FL., August 29-31, 2006.

10. Idaho National Laboratory, National Security, Jan. 2006 Need to Know newsletter,

http://www.inl.gov/nationalsecurity/newsletter/docs/jan2006.pdf. 